\title{
Notes on Factoring.
}

By J. W. Butters.

$\$ 1$. When it is necessary to find the factors of such an expression as $x^{2}-7 x-120$ the difficulty for beginners lies in finding the pair of factors of 120 which satisfy the middle term : in this case so that their difference is 7 . They may write down the pairs in order $(1 \times 120 ; 2 \times 60$; etc.) and then choose the suitable pair, but this method is often long; or they may guess until they chance to find the pair required. This is often done in so haphazard a fashion that much time is wasted, especially if the given expression have no rational factors.

\$2. A combination of these methods may be userl as follows: Take any pair of factors as a first trial-say $10 \times 12$; here the difference is too small; hence the required factors must differ more than 10 and 12 , and therefore the smaller factor must be made still smaller. Try now in order the natural numbers less than 10. We find that 9 is not a factor of 120 , but that 8 is (for $120=8 \times 15$ ) and that this gives the required difference. Hence

$$
x^{2}-7 x-120=(x+8)(x-15) .
$$

$\$ 3$. It sometimes happens that the first trial gives a sum or difference which differs greatly from the one required, and then the above method is rather long. For example, if for $x^{2}+59 x+168$ we take $8 \times 21$ as a first trial, we get in succession $7 \times 24$; $6 \times 28 ; 4 \times 42$; and $3 \times 56$ which is the pair required. In such a case we may begin by removing a factor from one of the pair and multiplying it into the other. We should then have (say) $8 \times 21$; $4 \times 42 ; 3 \times 56$. It ought to be noticed, however, that by this method we often pass the required pair. Thus we might have (by transferring 4 instead of 2) $8 \times 21 ; 2 \times 84$ where the first pair has too small a sum and the second too great. In most cases the method of $\S 2$ is sufficient, and if in using it we find two consecutive pairs with their (algebraic) sums too great and too small respectively, we see that the given expression cannot have rational factors.

34. Just as from the identity

$$
(x+a)(x+b) \equiv a^{2}+(a+b) x+a b
$$


we know that an expression of the form $x^{2}+p x+q$ may be resolved into factors provided we can find two factors of $q$ whose sum is $p$, so we may extend this method * to the form $A x^{2}+B x+C$ by means of the identity

$$
(a x+m)(b x+n) \equiv a b x^{2}+(a n+b m z) x+m n .
$$

Here we see that it is necessary to find two factors of $\mathrm{AC}$ whose sum is $\mathbf{B}$ (viz., an and $b m$ ); also that the required factors are obtained by removing $a$ and $b$ from the expressions $(a b x+a n)$ and $\left(a b x^{\prime}+b m\right)$, viz., $(b x+n)(a x+m)$.

$\$ 5$. One or two numerical examples will show how this works out in practice.

Find the factors of $18 x^{2}-111 x+80$.

Begin by writing down the product $18 \times 80$. [It is convenient to keep throughout the smaller number first.] This gives a sum of 98 instead of 111 . Hence 18 must be reduced. Applying now the method of natural numbers as in $\$ 2$ we find $16 \times 90$ (sum $=106$, too small) and $15 \times 96$ (sum 111, as required). Affix the proper signs : $-15 \times-96$. Now divide $(18 x-15),(18 x-96)$ by 3 and 6 (the G C.M.'s of 18 and 15 and 18 and 96 respectively) and we get $(6 x-5)(3 x-16)$ as the required factors.

\$6. In the following the necessary work alone is given:

$$
\begin{array}{l|c}
12 x^{2}-5 x-72 & 12 \times 72 \quad(\mathrm{~A}) \\
\equiv(4 x+9)(3 x-8) & 24 \times 36 \\
& +27 \times-32
\end{array}
$$

[Explanation: (A) The difference between 12 and 72 being very much greater than 5 , the factor 2 is transferred from 72 to 12 before applying the method of the natural numbers as in $\$ 2$. form.

\$7. This example may be used to present the theory in another

$$
\begin{aligned}
& 12 x^{2}-5 x-72 \equiv \frac{1}{12}\left\{(12 x)^{2}-5(12 x)-12 \times 72\right\} \\
\equiv & \frac{1}{12}(12 x+27)(12 x-32) \equiv \frac{1}{3}(12 x+27) \cdot \frac{1}{4}(12 x-32) \\
\equiv & (4 x+9)(3 x-8)
\end{aligned}
$$

- The extension is due to Mr Jae. M'Kean, F E.I.S., Leoturer on Mathe. matics in the Heriot-Watt College, Edinburgb. 
Since "factoring" is eminently a practical part of Algebra it is perhaps better not to burden the working of each case with the exemplification of the theory but to treat all as in $\S 6$.

$\$ 8$. This form of the theory suggests that instead of transforming quadratic equations from $a x^{2}+b x+c=0$ to $x^{2}+\frac{b}{a} x+\frac{c}{a}=0$, it is better to change to $(a x)^{2}+b(a x)+a c=0$. We thus avoid fractions and introduce early the important notion of "change of variable."

Against a Current Pseudo-Definition of Varying Velocity.

By Mr R. F. Muirhead 